\title{
Corrigendum
}

\section{Parameters predicting lymph node metastasis in patients with superficial esophageal squamous cell carcinoma}

Liyan Xue ${ }^{1}$, Liqun Ren ${ }^{1,2}$, Shuangmei Zou ${ }^{1}$, Ling Shan ${ }^{1}$, Xiuyun Liu $^{1}$, Yongqiang Xie ${ }^{1}$, Yueming Zhang ${ }^{3}$, Jun Lu ${ }^{1,4}$, Dongmei Lin ${ }^{1}$, Sanford M Dawsey ${ }^{5}$, Guiqi Wang ${ }^{3}$ and Ning Lu ${ }^{1}$

${ }^{1}$ Department of Pathology, Peking Union Medical College, Chinese Academy of Medical Sciences, Beijing, China; ${ }^{2}$ Department of Pathology, Chengde Medical College, Chengde, China: ${ }^{3}$ Department of Endoscopy, Cancer Institute (Hospital), Peking Union Medical College, Chinese Academy of Medical Sciences, Beijing, China; ${ }^{4}$ Department of Pathology, Beijing Chaoyang Hospital, Beijing, China and ${ }^{5}$ Nutritional Epidemiology Branch, Division of Cancer Epidemiology and Genetics, National Cancer Institute, Bethesda, MD, USA

Modern Pathology (2015) 28, 161; doi:10.1038/modpathol.2014.133

Correction to: Modern Pathology (2012) 25, 1364-1377; doi:10.1038/modpathol.2012.89; published online 25 May 2012.

In this paper, affiliation 1 is incorrect. The correct affiliation is 'Department of Pathology, Cancer
Institute (Hospital), Peking Union Medical College, Chinese Academy of Medical Sciences, Beijing, China.' 